\title{
Correction to: An Enhanced Meta-model to Generate Web Forms for Ontology Population
}

Petko Rutesic, Mirjana Radonjic-Simic, and Dennis Pfisterer $\mathbb{D}$

\author{
Correction to: \\ Chapter "An Enhanced Meta-model to Generate \\ Web Forms for Ontology Population" \\ in: B. Villazón-Terrazas et al. (Eds.): Knowledge Graphs \\ and Semantic Web, CCIS 1459, \\ https://doi.org/10.1007/978-3-030-91305-2_9
}

In the originally published version of chapter 9 the two references were incomplete.

The references [5] and [9] have been extended in the revised version. 